\title{
LA TERCERA MISION DE LAS UNIVERSIDADES Y SU APORTE POTENCIAL AL DESARROLLO NACIONAL
}

\author{
${ }^{1}$ Rubén Gómez Sánchez Soto. \\ ${ }^{1}$ Universidad Nacional de Ingeniería, Lima, Perú
}

Recibido: 05/09/2021 Revisado: 03/10/2021 Aceptado: 16/11/2021 Publicado: 30/01/2022

\section{RESUMEN}

El desempeño de las Universidades peruanas presenta mejoras importantes a partir de la aprobación de la Ley Universitaria 30220 promulgada el 03 de julio de 2014. Este dispositivo a su vez generó la creación de SUNEDU desde el 2015. Las mejoras alcanzadas pueden comprobarse por el licenciamiento, acreditación y producción científica. Es claro que los avances alcanzados luego de siete años no serían suficientes, para cerrar las brechas respecto de las Universidades ubicadas en las cincuenta primeras posiciones de los rankings internacionales; por ejemplo, (TOPUNIVERSITIES, 2021), y los niveles de desarrollo de los once (11) países donde están ubicadas tales universidades. La investigación ha permitido visualizar la urgencia de mejoras en la gestión de las universidades peruanas; particularmente, las públicas requieren iniciar proyectos de para mejorar su aporte al desarrollo nacional, ubicarse entre las universidades de tercera y cuarta generación. Análisis y reflexión, para pasar de la primer a la segunda y tercera misión y con esto enrumbar y aportar al liderazgo de la solución y priorización del desarrollo nacional, y no solo quedarse como protagonista de la problemática actual de las Universidades públicas.

Palabras claves: Tercera y cuarta generación, Tercera Misión, Universidades, Desarrollo Nacional, Investigación, innovación

\section{ABSTRACT}

The performance of Peruvian universities presents important improvements as of the approval of University Law 30220 promulgated on July 3, 2014. This device in turn generated the creation of SUNEDU since 2015. The improvements achieved can be verified by licensing, accreditation, and scientific production. The progress made after seven years would not be enough to close the gaps with respect to the Universities located 
in the top fifty positions in the international rankings; for example, (TOPUNIVERSITIES, 2021), and the levels of development of the eleven (11) countries where such universities are located.

The research has made it possible to visualize the urgency of improvements in the management of Peruvian universities; In particular, the public ones need to start projects to improve their contribution to national development, place themselves among the third and fourth generation universities. Analysis and reflection, to go from the first to the second and third mission and with this to direct and contribute to the leadership of the solution and prioritization of national development, and not only remain as the protagonist of the current problems of public universities.

Keywords: Third and fourth generation, Third Mission, Universities, National Development, Research, innovation

\section{INTRODUCCIÓN}

La problemática de las universidades peruanas, y particularmente las públicas se caracterizada por la necesidad de mejorar su aporte al desarrollo nacional, y para crear el circulo vicioso la necesidad de mayores presupuestos.

A cualquier ciudadano no le sería fácil comprender y estar de acuerdo con incrementos presupuestales, sin conocer el aporte resultante; esto sustenta, la necesidad de desarrollar un plan de mejora de la gestión de las Universidades Publicas de largo aliento y traspase varias gestiones de gobiernos. Se espera que este ensayo ayude a ese entendimiento.

\section{MATERIALES Y METODOS}

\section{Motivación para desarrollar la investigación}

El Perú como Nación no presenta una convergencia de ideas, objetivos, estrategias, y, en resumen, no se comparte una visión de futuro, entre sus principales involucrados. A continuación, se presentan la visión de las principales instituciones del Perú.

\section{Poder Legislativo Visión}

El Congreso expresa por excelencia la soberanía y pluralidad política de la República, en base al desempeño eficaz, honesto, legítimo y transparente de sus funciones de 
representación, legislación y control político, para afirmar sostenidamente la estabilidad democrática y la gobernabilidad del país, coadyuvando al bienestar de todos los peruanos.

Fuente: https://www.congreso.gob.pe/VisionMision/

\section{Poder Judicial Visión}

Administrar Justicia a través de sus órganos jurisdiccionales, con arreglo a la Constitución y a las leyes, garantizando la seguridad jurídica y la tutela jurisdiccional, para contribuir al estado de derecho, al mantenimiento de la paz social y al desarrollo nacional.

Fuente: https://www.pj.gob.pe/wps/wcm/connect/CorteSupremaPJ/s_Corte_Suprema/as_Conoc enos/mision_vision

\section{Universidad Nacional Mayor de San Marcos Visión}

Universidad del Perú, referente nacional e internacional en educación de calidad; basada en investigación humanística, científica y tecnológica, con excelencia académica; comprometida con el desarrollo humano y sostenible; líder en la promoción de la creación cultural y artística.

Fuente: http://previous.unmsm.edu.pe/home/inicio/vision

\section{Universidad Nacional La Agraria Visión}

La Universidad Nacional Agraria La Molina busca la excelencia y ser una institución líder, reconocida internacionalmente por su calidad académica por el impacto de sus investigaciones y por su contribución al desarrollo sostenible de la sociedad, en los sectores agrosilvopecuario, pesquero, alimentario y económico, educativo y social.

Fuente: http://www.lamolina.edu.pe/portada/acerca/mision_vision.htm

\section{Universidad Nacional de Ingeniería}

Institución académica con carreras profesionales acreditadas y reconocida internacionalmente por su alta calidad en investigación científica e innovación tecnológica, comprometida con el emprendimiento, la competitividad del capital humano en los ámbitos público y privado, con responsabilidad social, desarrollo sostenible y compromiso con el país.

Fuente: https://www.uni.edu.pe/index.php/institucion/misionyvision La falta de una orientación maestra (visión de futuro de consenso y socializada en la sociedad), muestra que las instituciones e incluso las tres principales universidades públicas de Perú, tienen orientaciones dispares, lo que dificulta el desarrollo de un aporte eficaz, oportuno, adecuado de la Academia para el desarrollo de la nación. Este escenario no permitiría una efectiva articulación de las universidades con la sociedad, y peor aún 
reflexionar sobre la pregunta ¿Cuáles deberían ser los aportes de la Academia al desarrollo de la Nación?

\section{Cambios y Avances en la Concepción de Misión de las Universidades}

Como consecuencia del análisis efectuado, y después de la investigación realizada sobre los cambios que se vienen desarrollando en la concepción de misión en las universidades de los países más avanzados, se tiene:

Por lo señalado, por (Jaeger, 2015) Las misiones convencionales de las Instituciones de Educación Superior (IES) comprenden, primera misión la generación y acumulación de conocimientos académicos, y la segunda misión la difusión de conocimientos a través de la educación académica.

Asimismo, según (Jaeger, 2015) La influencia de las IES en el desarrollo económico y social nacional y regional es un tema de especial interés y objeto prevalente de estudios y discusiones en las ciencias regionales. Y de acuerdo con (Jaeger, 2015) Especialmente en las economías basadas en el conocimiento, la calidad del sistema de educación superior es de gran importancia para el éxito del sistema nacional de innovación.

Para (Molas-Gallart et al., 2002) Muchos consideran que las universidades son fuentes cada vez más importantes de conocimientos y capacidades dentro de la economía del conocimiento.

De la misma forma (Molas-Gallart et al, 2002) señala: Tanto los formuladores de políticas como los analistas han comenzado a prestar más atención a las formas en que las capacidades y actividades universitarias pueden contribuir al desarrollo social y económico.

De acuerdo con (Jaeger, 2015) se considera que las IES son actores clave en los sistemas regionales de innovación, ya que ofrecen a las empresas regionales el potencial de transferencia de conocimiento y, por lo tanto, fomentan efectos de difusión de conocimiento localizados al enriquecer el escenario creativo de una región y proporcionar acceso continuo a instalaciones de investigación y capital humano altamente calificado. Los resultados señalados, ponen en valor el rol de las Universidades en su aporte al desarrollo y crecimiento económico; pero es preciso llevar a cabo las investigaciones para discernir y llegar a los modelos para canalizar los aportes. Tales avances serian fortalecidos con las simulaciones necesarias.

Conforme lo manifestado, se llega a la relación detallada en la figura 1. En esta figura se aprecia la importancia clave de la calidad del sistema de educación superior.

Figura 1

BIOTECH \& ENGINEERING Untels. Ene -Jun.2(1), 2022; ISSN:2788 -4295; 36-52 
Variables incidentes en el éxito de los Sistemas Nacionales de Innovación.

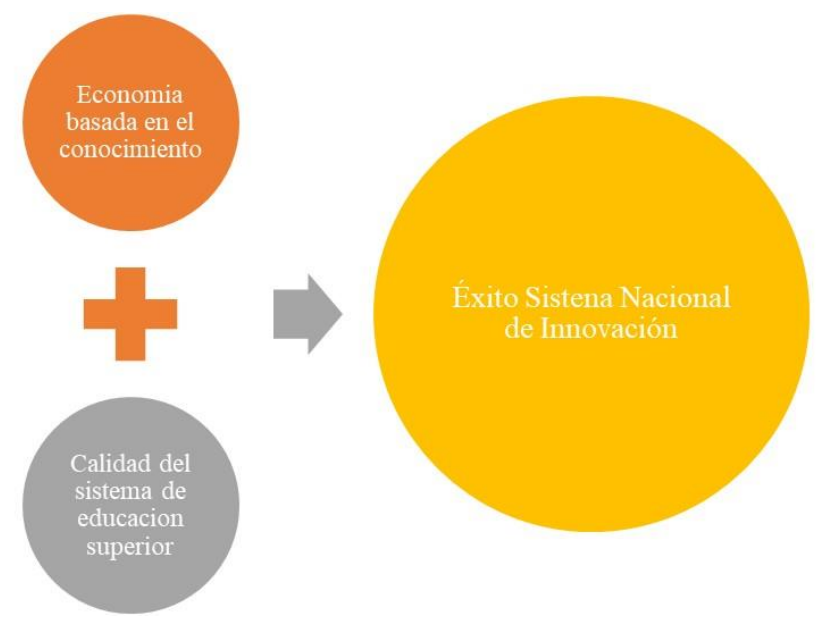

Fuente: Elaboración propia

Para (Molas-Gallart J. y Castro, 2007) La tercera misión de las universidades se refiere a todas las actividades relacionadas con la generación, uso, aplicación y explotación del conocimiento y otras capacidades universitarias fuera de los entornos académicos. Según (Ekonomiaz, 2017) La idea de la tercera misión ha ido ganando importancia en las dos últimas décadas, a medida que se anima a las universidades a poner en valor su labor investigadora en beneficio de la economía y la sociedad en general. Estas ideas consolidan la aplicación e importancia de la tercera misión de las Universidades.

Según (Molas-Gallart et al., 2002) El gobierno hace evidente sus orientaciones para condicionar la asignación presupuestal de las actividades de la Tercera Misión en una característica permanente del panorama de financiación universitaria. Se requiere que tales decisiones sobre el financiamiento se articulen con la información y resultados del desempeño de las actividades de la Tercera Misión.

\section{Universidades de Tercera y Cuarta Generación}

Según (Wissema, 2009), las universidades avanzan de una generación a otra. Hoy en día, la mayoría de las instituciones de educación superior tienen las características de las universidades de segunda generación y muchas están en transición a universidades de tercera generación. Igualmente, según (Wissema, 2009) Aun cuando el objetivo principal de las universidades de segunda generación es la educación y la investigación, el deber de las universidades de tercera generación incluye la tercera misión: crear y mantener alianzas con actores económicos fuera de la universidad, absorbiendo el conocimiento existente.

Sin embargo, es importante enfatizar que las instituciones de educación superior de hoy son mucho más heterogéneas de lo que sugiere la sistematización teórica de (Wissema, 
2009). Las líneas de orden no son tan nítidas y obvias, pero una serie de características de tercera generación están presentes en las instituciones de educación superior de hoy. Las principales universidades estadounidenses fueron las más rápidas en adaptarse a la nueva realidad, y Stanford y Silicon Valley personificaron la cooperación de los dos mundos de la ciencia y la economía que habían estado tan distantes en el pasado (Pawłowski, 2009).

(Pawłowski, 2009) presenta el concepto de "Universidad de Cuarta Generación", bajo el postulado que la universidad tradicional representa la primera generación; la universidad emprendedora, la segunda; y el conocimiento -subordinado, el tercero- en el que los tres elementos: educación, la investigación y los contactos con el entorno, son igualmente necesarios, y la universidad, a través de la transferencia de conocimientos, tecnologías y también de ideas, juega el papel de un artífice, cambiando su entorno cercano y lejano. La variable esencial de una universidad empresarial, al igual que la de la universidad de cuarta generación, es su modelo de gestión.

Dos factores caracterizan las economías de los países desarrollados: la globalización y el uso común del conocimiento como recurso vital, que son más importantes que las materias primas, las tecnologías o el capital y generan el mayor valor agregado (Pawłowski, 2009).

De igual forma, según (Pawłowski, 2009) no se puede pasar por alto que el PIB medio de los países que adoptaron modelos de gestión para administrar las universidades y en los que las universidades mejor articulación con el entorno social y económico (por ejemplo, Estados Unidos o Gran Bretaña) es superior a los países más grandes de Europa.

Según (Pawłowski, 2009) La mayoría de los países miembros de la Unión Europea asignan presupuestos para las actividades de todas las universidades públicas; la política distributiva considera el número de estudiantes, científicos y profesores.

Por lo explicado por (Pawłowski, 2009) En la Unión Europea emplea el criterio de "distribución" equitativa y democrática de los recursos presupuestarios, independientemente de la calidad del trabajo científico y resultados alcanzados.

De igual forma, según (Pawłowski, 2009) En los Estados Unidos; por el contrario, la mayoría de las universidades se financian con los pagos de los estudiantes; pero, las autoridades federales subvencionan la investigación en unas cincuenta mejores universidades de investigación. 
(Pawłowski, 2009) El porcentaje de fondos públicos destinados a I + D en la Unión Europea y en Estados Unidos es comparable (1\% y 1,2\%, respectivamente), la principal diferencia radica en el origen de los fondos:

- En la Unión Europea los fondos para I + D + I provienen de empresas e instituciones privadas.

- En Estados Unidos, el porcentaje de fondos privados asignados a I + D +I es el doble que en la UE.

Resaltan la rapidez con la que las universidades y las empresas relacionadas implementar los logros científicos. Esto es el resultado del modelo de gestión (Pawłowski, 2009).

(Lukovics \& Zuti, 2014) propusieron una clasificación sistemática de las cuatro generaciones de universidades (Tabla 1).

(Lukovics \& Zuti, 2014) describieron las claves de las universidades de cuarta generación de la siguiente manera: tanto la sociedad como la economía están posicionadas en una fase de globalización, y emplean intensamente las tecnologías de la información, donde el enfoque estratégico es una característica clave.

Similarmente (Lukovics \& Zuti, 2014) señala: Además de discutir las tres misiones principales, también está presente el desarrollo consciente y orientado al futuro de la economía local.

\section{Tabla 1}

Caracterización de las universidades de tercera y cuarta generación

\begin{tabular}{|c|c|c|c|c|}
\hline Aspecto & $\begin{array}{l}\text { Primera } \\
\text { generación de } \\
\text { Universidades }\end{array}$ & $\begin{array}{c}\text { Segunda } \\
\text { generación de } \\
\text { Universidades }\end{array}$ & $\begin{array}{c}\text { Tercera } \\
\text { generación de } \\
\text { Universidades }\end{array}$ & $\begin{array}{c}\text { Cuarta generación de } \\
\text { Universidades }\end{array}$ \\
\hline Objetivo & Educación & $\begin{array}{l}\text { Educación e } \\
\text { investigación }\end{array}$ & $\begin{array}{l}\text { Educación, } \\
\text { investigación y } \\
\text { utilización de } \\
\text { conocimiento }\end{array}$ & $\begin{array}{l}\text { Educación, investigación, } \\
\text { investigación y desarrollo } \\
\text { e innovación, utilización } \\
\text { del conocimiento y } \\
\text { desarrollo económico } \\
\text { proactivo }\end{array}$ \\
\hline Rol & $\begin{array}{l}\text { Protección de } \\
\text { la verdad }\end{array}$ & $\begin{array}{c}\text { El } \\
\text { conocimiento } \\
\text { de la } \\
\text { naturaleza }\end{array}$ & $\begin{array}{c}\text { Creación de } \\
\text { valor agregado }\end{array}$ & $\begin{array}{l}\text { Acelerador económico } \\
\text { local, estrategia de } \\
\text { determinación }\end{array}$ \\
\hline Entregables & Profesionales & $\begin{array}{l}\text { Profesionales } \\
\text { y científicos }\end{array}$ & $\begin{array}{l}\text { Profesionales, } \\
\text { científicos y } \\
\text { emprendedores. }\end{array}$ & $\begin{array}{l}\text { Profesionales, científicos, } \\
\text { emprendedores y } \\
\text { economía local } \\
\text { competitiva }\end{array}$ \\
\hline
\end{tabular}


Idiomas Latín Nacional Ingles $\begin{aligned} & \text { Multilingüe (nacional e } \\ & \text { inglés) }\end{aligned}$

$\begin{array}{llccl}\text { Gestión } & \begin{array}{c}\text { Rector de la } \\ \text { Universidad }\end{array} & \begin{array}{c}\text { Científicos a } \\ \text { tiempo parcial }\end{array} & \begin{array}{c}\text { Gestión } \\ \text { profesional }\end{array} & \begin{array}{l}\text { Gestión profesional y } \\ \text { expertos locales. }\end{array}\end{array}$

Fuente: (Lukovics \& Zuti, 2014)

\section{Impacto económico de las Universidades}

Según (Etzkowitz, 1998) Actualmente, las universidades están experimentando una "segunda revolución" en estos días, incorporando el desarrollo económico y social como parte de su misión. Ahora la universidad emprendedora emergente integra el desarrollo económico como una función adicional.

Según (Etzkowitz, 1998) El premio Nobel Joshua Lederberg encontró los problemas científicos y las recompensas financieras demasiado intrigantes como para no involucrarse.

De forma similar (Etzkowitz, 1998) señala: Otro Nobel, Arthur Kornberg, expresó su perplejidad en su autobiografía 'La hélice dorada' de que un científico académico altamente enfocado como él se hubiera convertido en un defensor de la relación industria academia, encontrándola fructífera tanto para la ciencia como para los negocios

La tercera misión de las universidades incluye 'todas las actividades relacionadas con la generación, uso, aplicación y explotación del conocimiento y otras capacidades universitarias fuera de los entornos académicos' (Molas-Gallart \& Castro-Martinez, 2007).

Las universidades, requieren orientar sus esfuerzos para lograr implementar su tercera misión; estas necesidades requieren del acompañamiento de políticas claras. Mientras que existe un interés académico comprensible en el impacto de las iniciativas de participación social y empresarial en el carácter de la actividad universitaria y la investigación financiada con fondos públicos (Molas-Gallart \& Castro-Martinez, 2007).

Según (Molas-Gallart \& Castro-Martinez, 2007) Además, las universidades hacen contribuciones al gobierno y a la sociedad civil, así como al sector privado, ayudar no solo con el desempeño económico, sino también ayudar a mejorar la calidad de vida y la eficacia de los servicios públicos.

Durante al menos un par de décadas, los gobiernos de muchos países europeos se han preocupado por la forma en que las universidades y los establecimientos públicos de investigación se relacionan con la sociedad (Molas-Gallart \& Castro-Martinez, 2007). 
Según (Molas-Gallart \& Castro-Martinez, 2007) Se han lanzado muchas iniciativas diferentes para incrementar el uso fuera de los entornos académicos de las capacidades que residen dentro de las organizaciones universitarias.

En términos generales y amplios: incrementar la contribución de las universidades a la economía y la sociedad es un objetivo general que se repite en todos los países (MolasGallart \& Castro-Martinez, 2007). Este tipo de decisiones, requieren ser parte de las políticas públicas de cada país, y según (Molas-Gallart \& Castro-Martinez, 2007) Que este objetivo general pueda ser interpretado de manera diferente, o incluso divergente, queda para el desarrollo práctico y la aplicación de las intervenciones de política.

Según (Lukovics \& Zuti, 2014) ya que la competitividad no se basa en los recursos naturales y la fuerza de trabajo física, sino en la creación de conocimiento y el desarrollo continuo.

De acuerdo con (Lukovics \& Zuti, 2014) Las universidades comprometidas a nivel regional también dependen en gran medida de la integración de actividades educativas, de investigación y de tercera misión en la circulación de la economía de la región, creando así valor agregado.

De acuerdo (Molas-Gallart et al., 2002) nuestro marco analítico comienza con una distinción básica entre lo que las universidades tienen (capacidades) y lo que hacen (actividades):

- Según (Molas-Gallart et al., 2002) Las universidades de investigación tienen capacidades en dos áreas principales: (a) capacidades de conocimiento y (b) instalaciones físicas. Estas capacidades se desarrollan a medida que las universidades llevan a cabo sus funciones básicas de docencia e investigación.

- De la misma forma, según (Molas-Gallart et al., 2002) las universidades llevan a cabo tres conjuntos principales de ocupaciones; ellos: (1) enseñar, (2) investigación (3) comunicar los resultados de su trabajo. Todas estas actividades se pueden considerar tercera misión cuando involucran o se dirigen a comunidades no académicas.

Según (Molas-Gallart et al., 2002) La figura 2 presenta las actividades de la tercera misión organizadas. La figura muestra una definición amplia de la gama de actividades que dan forma e influyen en la relación entre las universidades y el resto de la sociedad

\section{Figura 2}

Marco conceptual para analizar la tercera misión 


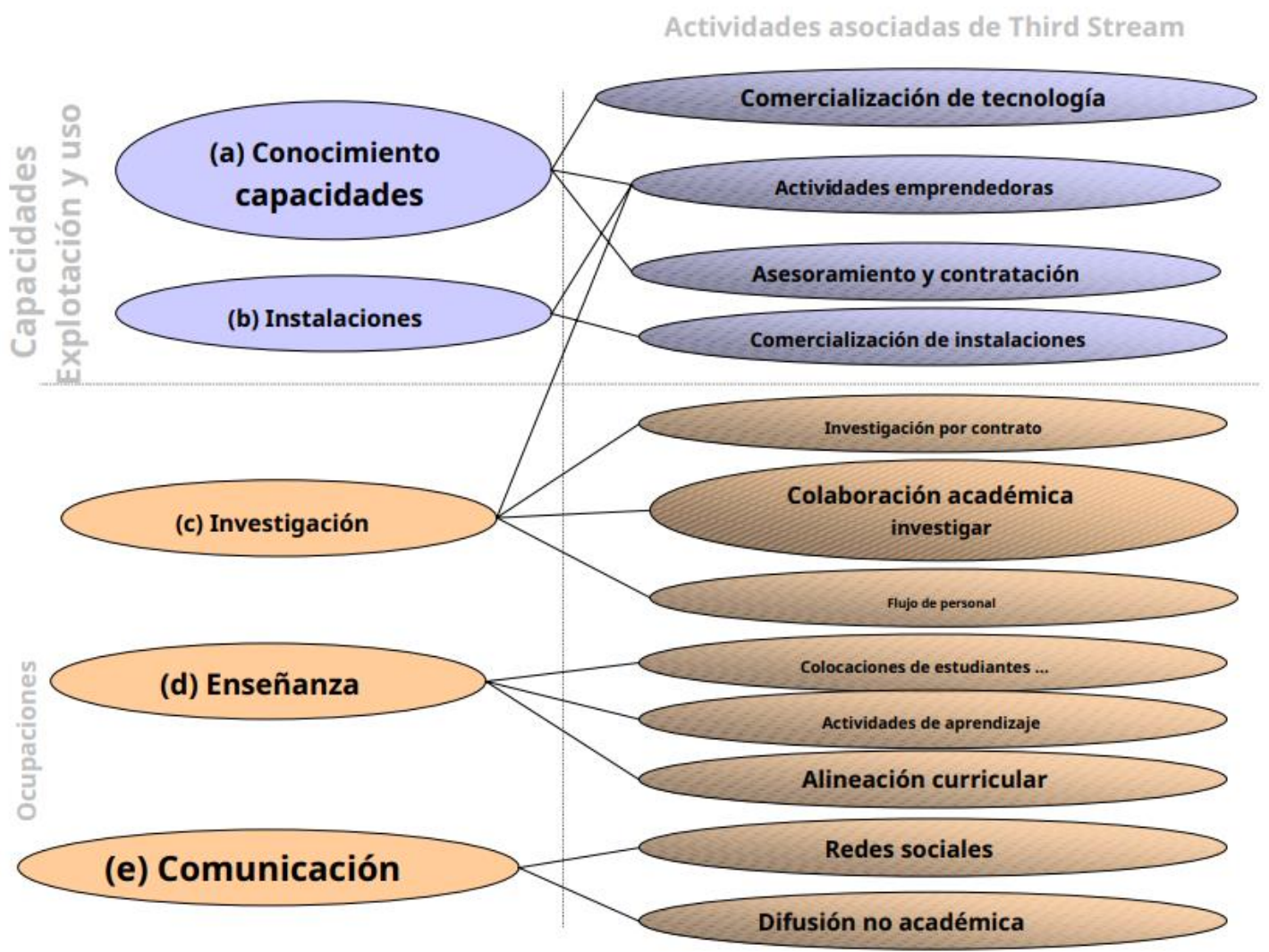

Fuente: (Molas-Gallart, 2002)

Según (Ekonomiaz, 2017) las características organizativas, incentivos y estructuras regulatorias también influyen en el porcentaje y tipo de interacciones industriauniversidad. En muchos países, por ejemplo, se han introducido cambios legislativos para desarrollar incentivos y condiciones marco para la explotación de la investigación académica.

Asimismo, según (Ekonomiaz, 2017) En otros se han introducido cambios en las políticas cuyo objetivo era promover la difusión y aplicación de la investigación con resultados diversos, como la Ley Bayh-Dole en EE. UU. o la abolición del llamado privilegio de los profesores.

Según (Ekonomiaz, 2017) La decisión y apoyo de políticas a la tercera misión de las universidades también ha dado lugar a una importante expansión de la infraestructura y capacidades de intercambio de conocimientos, incluidas la creación de oficinas de transferencia de tecnología y otras áreas como las incubadoras de empresas y los parques tecnológicos.

\section{Tabla 2}

Indicadores sugeridos para validación de tercera misión de las universidades 


\begin{tabular}{|c|c|}
\hline $\begin{array}{l}\text { Área / actividad } \\
\text { objetivo }\end{array}$ & Indicadores sugeridos \\
\hline \multirow{3}{*}{$\begin{array}{l}\text { Transferencia } \\
\text { tecnológica }\end{array}$} & $\begin{array}{l}\text { Ingresos obtenidos por la universidad por la utilización del capital } \\
\text { intelectual }\end{array}$ \\
\hline & $\begin{array}{l}\text { Número de contratos de investigación conjunta con empresas } \\
\text { innovadoras }\end{array}$ \\
\hline & $\begin{array}{l}\text { Número / tasa regional de empresas orientadas a la innovación } \\
\text { creadas en la universidad }\end{array}$ \\
\hline \multirow{10}{*}{ Asesoramiento } & Número de estrategias de desarrollo económico creadas \\
\hline & Número de empresas que recurren a los servicios de asesoramiento \\
\hline & $\begin{array}{l}\text { Porcentaje de empresas universitarias spin-off / start-up en la } \\
\text { aglomeración }\end{array}$ \\
\hline & $\begin{array}{l}\text { El número de spin-offs / start-ups por cada } 1000 \text { empleados } \\
\text { universitarios }\end{array}$ \\
\hline & El número de spin-offs / start-ups creadas en los últimos 5 años. \\
\hline & Los ingresos de spin-offs / start-ups \\
\hline & $\begin{array}{l}\text { El número de empleados de las spin-offs / start-ups en los últimos } 5 \\
\text { años. }\end{array}$ \\
\hline & $\begin{array}{l}\text { El número de empresas creadas por estudiantes o graduados de los } \\
\text { últimos } 5 \text { años. }\end{array}$ \\
\hline & Número de conexiones de I + D industrial \\
\hline & Número de agentes de $\mathrm{I}+\mathrm{D}$ \\
\hline Universidad - & $\begin{array}{l}\text { Número de proyectos conjuntos realizados por universidad e } \\
\text { industria en los últimos } 5 \text { años }\end{array}$ \\
\hline \multirow[t]{2}{*}{$\begin{array}{l}\text { Gobierno } \\
\text { relaciones }\end{array}$} & $\begin{array}{l}\text { Porcentaje de empresas innovadoras (como porcentaje de todas las } \\
\text { empresas) que cooperan con la universidad }\end{array}$ \\
\hline & $\begin{array}{l}\text { Porcentaje de actividades de I }+ \text { D universitarias financiadas por la } \\
\text { industria }\end{array}$ \\
\hline Comercialización de & Ingresos por alquiler (por ejemplo, laboratorios) \\
\hline $\begin{array}{l}\text { instalaciones } \\
\text { académicas }\end{array}$ & Número de eventos públicos organizados por la universidad \\
\hline Mejora de la & Numero de eventos culturales \\
\hline
\end{tabular}


compromiso social de Número de visitantes internos

la Universidad

Número de visitantes externos

Número de comunicados de prensa en un período de tiempo determinado (personal

universitario, investigadores en medios regionales y nacionales)

Número de eventos universitarios que promueven la responsabilidad social (por ejemplo, programas ecológicos)

Número de programas de difusión (ciencia al público en general)

Fuente (Lukovics y otros, 2016)

\section{Estatus de la Universidad Peruana}

De acuerdo con (Benites, 2021) el Perú ha experimentado un proceso de masificación de la educación universitaria. Este se define como un proceso en el que los estados buscan que la mayoría de su población se involucre en alguna forma de educación superior.

Según (Benites, 2021) La masificación puede verse con claridad en el incremento del porcentaje total de las y los jóvenes (de 17 a 24 años) matriculados en educación universitaria.

Por lo señalado por (Benites, 2021) La figura 3 muestra un importante crecimiento: de 426 mil 029 estudiantes matriculados en el 2000 (10.4\%) a 1 millón 206 mil 137 en el $2019(26.4 \%)$

\section{Figura 3}

Porcentaje de matriculados en educacion universitaria 2000-2020

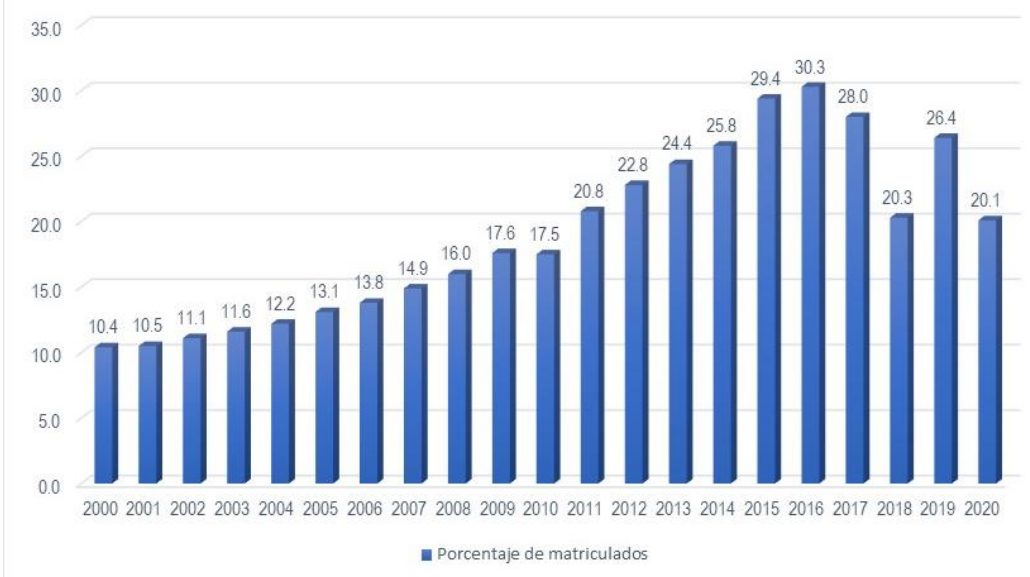

Nota. Elaboración propia sobre la base de ANR (2000-2012), INEI (2011-2018) y Ministerio de educación $(2019,2020)$

Fuente: (Benites, 2021) 
Según (Benites, 2021) En el año 2000, las universidades públicas concentraban el 59,8\% del total de estudiantes universitarios; mientras que, en el año 2019, este valor se redujo a 16,2\%. De igual forma, según (Benites, 2021) Desde el 2006 en adelante, las universidades privadas se convirtieron en el principal proveedor de educación universitaria en el país. Hoy, 8 de cada 10 universitarios estudia en una universidad privada.

\section{Figura 4}

Porcentaje del total de matriculados en educación universitaria, 2000-2020

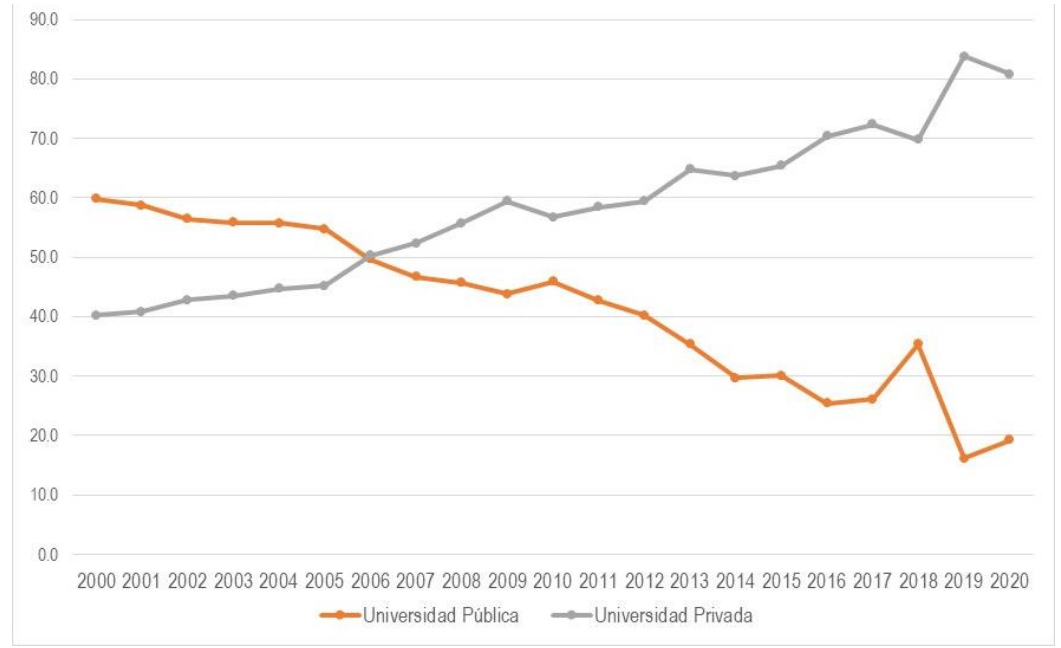

Nota. Elaboración propia sobre la base de ANR (2000-2012), INEI (2011-2018) y Ministerio de educación $(2019,2020)$

Fuente: (Benites, 2021)

\section{RESULTADOS}

Por las investigaciones realizadas, respecto de los cambios verificados en la gestión de las universidades, respecto de los avances en sus modelos de gestión, para alcanzar la categorización de tercera o cuarta generación, se requiere anotar las siguientes:

a. Los países desarrollados han aplicados diferentes políticas públicas en temas de gobernabilidad y decisiones; por ejemplo, sobre cómo financiar los presupuestos de las Universidades, y cómo financiar la investigación en general. Los resultados alcanzados por las Universidades de Europa y Estados Unidos lo hacen evidente.

b. La generación de conocimiento es clave para el crecimiento económico de los países. Los resultados que se viene alcanzando en los países desarrollados tiene estrecha relación con el hecho de contar universidades de tercera y cuarta 
generación. Esto demuestra que se requiere una relación clara y sostenida de las Universidades con las empresas y sociedad.

c. Según el ranking internacional (TOPUNIVERSITIES, 2021) hay Universidades peruanas consideradas en las siguientes posiciones: 432 Pontificia Universidad Católica del Perú, 701-750 Universidad Peruana Cayetano Heredia, 801-1000 Universidad Nacional Mayor de San Marcos, en más de 1000 Universidad de Lima, Universidad del Pacifico, Universidad Nacional Agraria La Molina, Universidad Peruana de Ciencias Aplicadas. Es necesario señalar que el Instituto de Tecnología de Massachusetts (MIT) mantiene el primer lugar en el este mismo ranking internacional durante nueve años. De las siete universidades ubicadas en el este ranking internacional solo dos son universidades públicas.

d. Es claro que en el Perú a pesar de existir una Visión al 2050, discutida y desarrollada por el Acuerdo Nacional y CEPLAN no está arraigada en el país. Esta es una problemática de base; ya que los esfuerzos, de los principales involucrados no tiene una orientación clave y estrategia para optimizar las inversiones, políticas y decisiones en cuanto a desarrollo nacional. Las Universidades peruanas, y primordialmente las Universidad Publicas, requieren asumir compromisos del más alto nivel para iniciar los cambios en sus modelos de gestión y enrumbar hacia proyectos que les permitan ser calificadas como de tercera y cuarta generación,

e. Los resultados alcanzados por (Benites, 2021) son más que una alerta. Por un lado, indica que entre el 2000 al 2019 ha habido un incremento de 780,108 de estudiante matriculados en las Universidades peruanas, esto sería un buen indicativo; pero, los estudiantes matriculados en las universidades públicas han disminuido de $80 \%$ de matriculados a un $20 \%$ de matriculados entre los años 2000 al 2020, esto significa una caída del $60 \%$ de matriculados. Y como contraparte las matrículas en las universidades privadas para esos mismos años se han incrementado de $40 \%$ al 80\%; lo que, significa que 8 de cada 10 universitarios estudia en una universidad privada. Las figuras 3 y 4 sustentan los comentarios.

f. Las posiciones de las universidades peruanas en el ranking internacional (TOPUNIVERSITIES, 2021), y los resultados de (Benites, 2021), muestran la necesidad de que el Sistema Educativo Superior efectúe un análisis importante y reflexión para identificar las mejoras que se requieren para lograr universidades bajo el calificativo de Tercera Misión. Pero esto necesitaría el acompañamiento y 
liderazgo de políticas nacionales priorizadas, ya que tiene que ver con el futuro y desarrollo nacional del país.

g. Según las investigaciones y los resultados de (Lukovics \& Zuti, 2014) detallados en la Tabla 1, de (Molas-Gallart, 2002) mostrados en la Figura 2, y la lista de indicadores sugeridos para validación de la tercera misión de las Universidades, según (Lukovics y otros, 2016) se dispone de la información de base para esbozar los lineamientos del proyecto de investigación para iniciar los trabajos y generar que las Universidades se orienten a ser catalogadas como de Tercera Misión.

\section{DISCUSION}

Los resultados señalados por (Benites, 2021) en cuanto a las variaciones de matriculados en las universidades públicas y privadas. Y, por otro lado, las ubicaciones alcanzadas por las universidades peruanas, en el ranking internacional (TOPUNIVERSITIES, 2021), genera una problemática, pero también origina la discusión sobre ¿por dónde empezar para atacar la problemática? Ante esta reflexión, se entiende que prima la motivación para desarrollar el presente ensayo: "La tercera misión de las universidades y su aporte potencial al desarrollo nacional"; por lo que, entonces se requiere priorizar el aporte de las Universidades al desarrollo nacional. Bajo esta premisa se recomendarán; por lo menos, tres líneas de acción:

a. Sería necesario que las Universidades públicas puedan priorizar la conformación de grupos de investigación que tomen las ideas detalladas en el inciso "g" de los resultados de la presente investigación.

b. De forma similar las Universidades públicas tendrían que priorizar sus políticas, para definir los ajustes en sus modelos de gestión y se logren demostrar que van alcanzando los hitos de la Tercera Misión. Es evidente que la investigación requiere ser la clave de la formación profesional en pre y posgrado.

c. Las universidades públicas requerirán implementar dos estudios: en primer lugar, un estudio de posicionamiento y líneas base. Este se convertiría en la hoja de ruta para los cambios y ajustes en general en el ámbito del desarrollo y gestión universitaria. Y posteriormente, un estudio de futuro de las universidades; en el cual se priorizaría la aplicación de la prospectiva y construcción de futuro, para 
orientar el modelo educativo a implementar en los próximos veinticinco a treinta años.

\section{CONCLUSIONES}

El presente ensayo ha permitido poner sobre la mesa, una serie de resultados de investigaciones y logros de universidades de los países desarrollados como tema de análisis y benchmarking respecto de las universidades peruanas y particularmente de las universidades públicas. Las acciones por desarrollar en las Universidades publicas su8geridas por los resultados y discusión del presente ensayo necesita de una priorización por las personas que lideran tales universidades, y funcionarios que forman parte del poder y ejercen la gobernanza del país.

\section{POSIBLES LIMITACIONES DEL PROYECTO}

La principal limitación es que el ensayo no llegue a los niveles de las personas que tomen decisiones sobre la gestión de las universidades y de los niveles de decisiones de política nacional.

\section{REFERENCIAS}

Benites, R. (Abril de 2021). La Educación Superior Universitaria en el Perú postpandemia. Lima, Lima, Perú.

Ekonomiaz. (2017). Papel de la universidad en el desarrollo regional. Revista Vasca de Economia, II $(92)$.

Etzkowitz, H. (1998). Las normas de la ciencia empresarial: efectos cognitivos de las nuevos vínculos universidad-industria. Elsevier, Politica de investigacion(27), 823-833.

Jaeger. (2015). Medición del "potencial de tercera misión" regional de diferentes tipos de IES. NIERS.

Lukovics, M., \& Zuti, B. (2014). Universities enhancing regional competitiveness: "fourth generation" universities? Space and Society, 77-96. 
Lukovics, M., Kotosz, B., Molnár, G., \& Zuti, B. (2016). How to Measure the Local Economic Impact of Universities? Methodological Overview. MPRA, 5(2), 3-19. https://doi.org/DOI: 10.15196/RS05201

Molas-Gallart. (2002). Final Report to the Russell Group of Universities. SPRU(April), 2-98.

Molas-Gallart, J., \& Castro-Martinez, E. (2007). Ambiguity and conflict in the development of "Third Mission" indicators. Valencia, Valencia, España.

Pawłowski, K. (2009). The 'Fourth Generation University' as a Creator of the Local and Regional Development. Higher Education in Europa, 34, 51-64.

TOPUNIVERSITIES, Q (2021). Q. QS TOPUNIVERSITIES. https://www.topuniversities.com/university-rankings/world-universityrankings/2021

Wissema, J. (2009). Toward the Third Generation University Managing the University in Transition. Edward Elgar Publishing Limited. 